Figure S1

\title{
Diameter-light relationship
}

(A) Light estimate vs. tree diameter

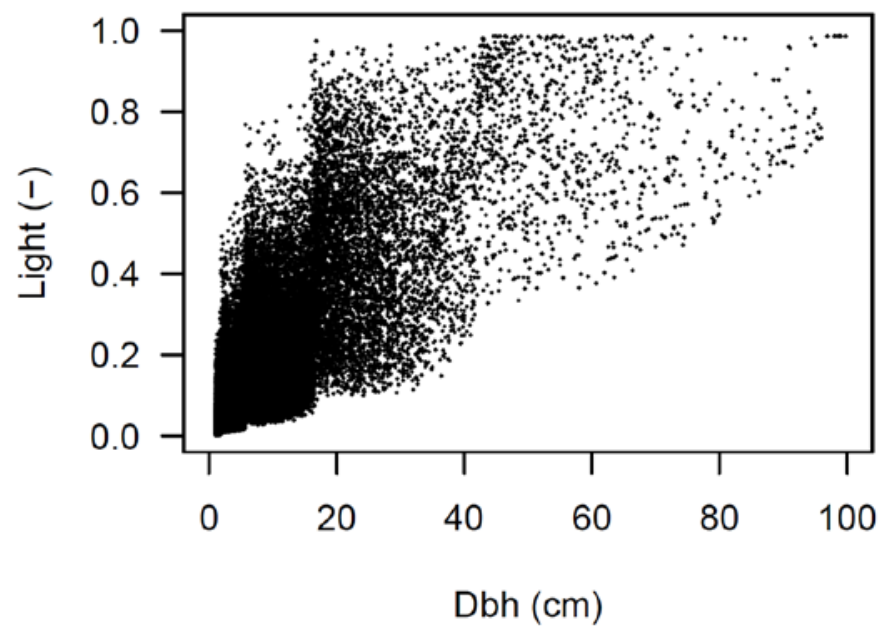

Light estimate vs. tree diameter (dbh) for 148933 trees at Barro Colorado Island, Panama, in 1990. Trees with $\mathrm{dbh}>1 \mathrm{~m}$ are assumed to receive full sunlight and are not shown.

(B) Nonlinear regression estimating average tree dbh for light levels between 2 and 20\%

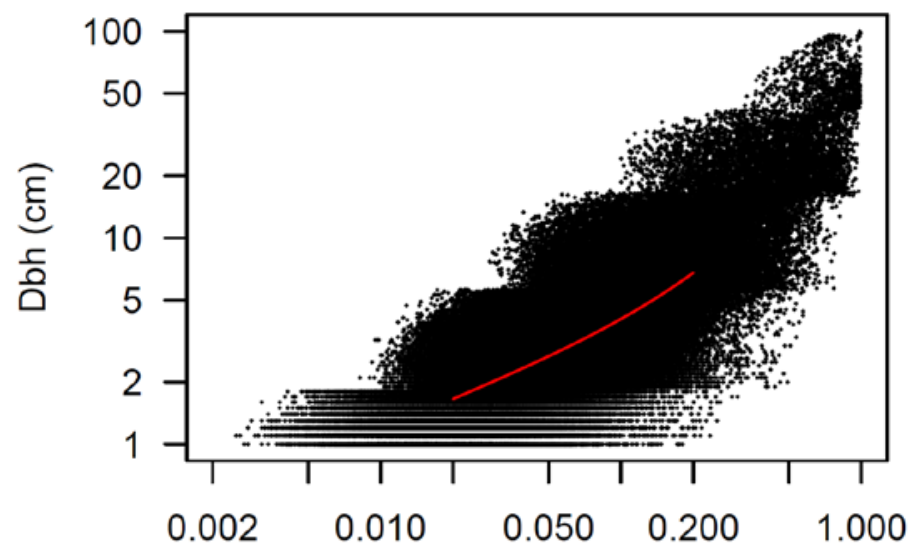

Nonlinear regression predicting average $\log (\mathrm{dbh})$ in the light range from 2 to $20 \%(\log (d b h)=4.547+$ $0.455 \log ($ light $)+2.006$ light; $d$ bh is in $\mathrm{mm}$ ). Average $\log (\mathrm{dbh})$ is used to estimate average growth across the light range for Fig. 4 of the main manuscript.

Light (-) 